\title{
Development of the Hard-X-ray Angle Resolved X-ray Photoemission Spectrometer for Laboratory Use
}

\author{
Masaaki Kobata, ${ }^{* 1 \dagger}$ Igor Píšs, ${ }^{* 1, * 2}$ Hideo IwaI, ${ }^{* 3}$ Hiromichi YAMazuI, ${ }^{* 4}$ Hiroaki TaKaHaSHI, ${ }^{* 4}$ \\ Mineharu SuzukI, ${ }^{* 4}$ Hiroyuki Matsuda, ${ }^{* 5}$ Hiroshi DaIMon, ${ }^{* 5}$ and Keisuke KobaYaSHI ${ }^{* 1}$ \\ *1 National Institute for Materials Science (NIMS), SPring-8 BL15XU, 1-1-1 Koto, Sayo-cho, Sayo, \\ Hyogo 679-5148, Japan \\ *2 Department of Surface and Plasma Science, Faculty of Mathematics and Physics, Charles University in Prague, \\ 18000 Prague 8, Czech Republic \\ *3 National Institute for Materials Science (NIMS), 1-2-1 Sengen, Tsukuba, Ibaraki 305-0047, Japan \\ *4 ULVAC-PHI, Inc., 370 Enzo, Chigasaki, Kanagawa 253-8522, Japan \\ *5 Graduate School of Materials Science, Nara Institute of Science and Technology (NAIST), \\ 8916-5 Takayama, Ikoma, Nara 630-0192, Japan
}

\begin{abstract}
This article reports development of a practical laboratory hard X-ray photoelectron spectroscopy (HXPS) system by combining a focused monochromatic $\mathrm{Cr}_{\alpha} \mathrm{X}$-ray source, a wide angle acceptance objective lens and a high kinetic energy electron analyzer. The $\mathrm{Cr} \mathrm{K}_{\alpha}$ source consists of a $\mathrm{Cr}$ target, a $15 \mathrm{kV}$ focused electron gun, and a compact bent crystal monochromator. The X-ray spot size is variable from $10 \mu \mathrm{m}(1.25 \mathrm{~W})$ to $200 \mu \mathrm{m}(50 \mathrm{~W})$. A wide acceptance angle objective lens is installed in front of a hemispherical analyzer. The total resolution of $0.53 \mathrm{eV}$ was obtained by Au Fermi-edge measurements. Angle acceptance of $\pm 35^{\circ}$ with angle resolution of $0.5^{\circ}$ was achieved by measuring $\mathrm{Au} 3 \mathrm{~d}_{5 / 2}$ peak through a hemicylinder multi-slit on an Au thin strip, in angle resolution mode. As the examples, silicon based multilayer thin films were used for showing the possibilities of deeper (larger) detection depth with the designed system.
\end{abstract}

(Received September 18, 2009; Accepted December 11, 2009; Published February 10, 2010)

\section{Introduction}

A practical throughput with an adequate performance is our most important target for the development of a hard X-ray photoelectron spectroscopy (abbreviations HAXPES, HX-PES, or HXPES have been used in the literature, however, here we use HXPS, which ties well to SXPS, the abbreviation for soft $\mathrm{X}$-ray photoemission spectroscopy) laboratory system. If this is realized, it is expected to be very effective for offering the opportunities of bulk sensitive HXPS applications to users widespread in various fields of science and technology. ${ }^{1}$ The first trial of HXPS with high throughput and high-energy resolution for core level and valence band studies using high energy and high-brilliance synchrotron radiation has been successfully performed at SPring- $8 .^{2}$ High energy excitation results in larger probing depths of photoelectrons compared to conventional PES, and enabled a study of bulk-sensitive electronic properties of materials in actual device structures much less influenced by surface conditions. ${ }^{1-3}$ With this technique, requirements for surface preparation are greatly reduced, if not eliminated. ${ }^{4}$ It is also possible to derive in-depth information of chemical state distribution of much deeper regions than those obtained by conventional $\mathrm{Al} \mathrm{K} / \mathrm{Mg} \mathrm{K}_{\alpha}$ sources, by measuring take-off-angle (TOA) dependence of core

$\dagger$ To whom correspondence should be addressed.

E-mail: m-kobata@spring8.or.jp level spectra.

The essential drawback of HXPS is that high-energy excitation is associated with rapid decrease in photo-ionization cross-section $(\sigma) .^{5}$ Consequently, HXPS has been realized in synchrotron radiation facilities, mostly been possible by using high-energy and high-brilliance undulator X-rays in the third generation synchrotron radiation facilities such as SPring-8 (Super Photon ring-8 GeV, Japan) and ESRF (European Synchrotron Radiation Facility, France) ${ }^{1}$

In order to realize the laboratory HXPS system with practical throughput, it is necessary to overcome the lack of signal intensities. In this paper we report the results of our development, achieved by devising a combination of a compact focused monochromatic $\mathrm{Cr} \mathrm{K}_{\alpha} \mathrm{X}$-ray source, a wide acceptance objective lens, and a high energy hemispherical electron analyzer with large $(200 \mathrm{~mm})$ mean radius.

\section{System design and configuration}

Figure 1 shows the configuration of the laboratory HXPS system that we have developed. This system consists of a focused $\mathrm{Cr} \mathrm{K}_{\alpha}$ source, a wide acceptance objective lens, and a high energy version of VG Scienta R4000 analyzer. All the components are mounted on a bench, which is designed to be aligned easily.

One of the crucial keys in the laboratory HXPS system development is to devise a monochromatic X-ray source with high-flux and small spot size on a sample surface. The second requirement is very important to accept photoelectrons 


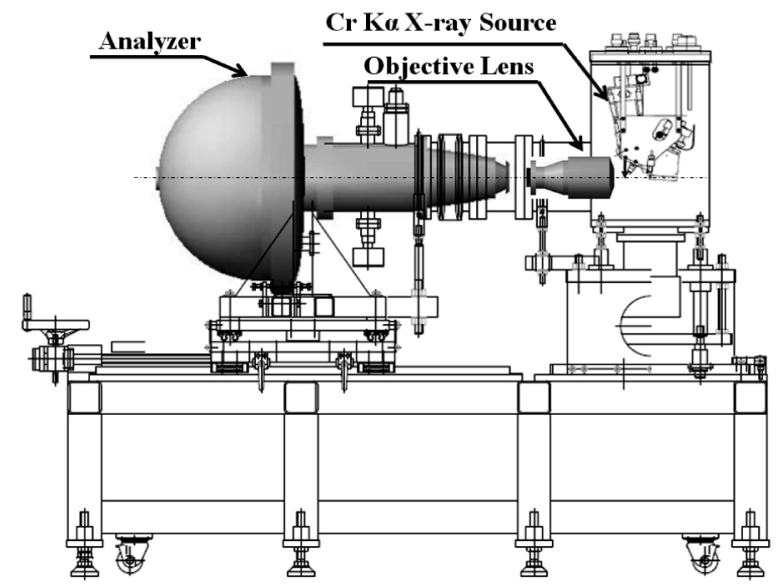

Fig. 1 Schematic view of laboratory HXPS combined with the wide acceptance angle objective lens, monochromatic $\mathrm{Cr} \mathrm{K}_{\alpha} \mathrm{X}$-rays, and VG Scienta R4000 $10 \mathrm{kV}$ analyzer.

effectively into the electron analyzer. We adopted an X-ray source design similar to that is used in VersaProbe (ULVAC-PHI, Inc.). The water cooled $\mathrm{Cr}$ target is bombarded by a focused electron beam. The emitted $\mathrm{Cr} \mathrm{K}_{\alpha}(5.4 \mathrm{keV}) \mathrm{X}$-rays are monochromatized, and focused onto a sample surface by a compact bent crystal monochromator with a $300 \mathrm{~mm}$ Roland circle as shown in Fig. 2(a). The X-ray beam can be scanned on the sample by moving the focused electron beam on the target. A minimum X-ray spot size of $9 \mu \mathrm{m} \times 8 \mu \mathrm{m}$ was achieved from the secondary electron image of a copper mesh grid, as shown in Fig. 2(b). The X-ray spot size is variable from $10 \mu \mathrm{m}$ $(1.25 \mathrm{~W})$ to $200 \mu \mathrm{m}(50 \mathrm{~W})$.

The development of a wide-acceptance objective lens with good angle-resolution capability is another crucial key. Matsuda and Daimon designed an objective lens to fulfill these specifications using an ellipsoidal mesh electrode in the first stage of the lens. ${ }^{6,7}$ The high-precision fabrication of an ellipsoidal metal mesh electrode in the first stage electrode of the lens is the most important point in this objective lens development. The specifications for the objective lens that we have developed are as follows: $\pm 45^{\circ}$ acceptance angle, $\pm 9^{\circ}$ exit angle, magnification factor of 5 , spot size at the exit point $0.5 \mathrm{~mm}$ or less, angular resolution of $0.5^{\circ}$, and maximum operating voltage at electrodes of $10 \mathrm{kV}$. Figure 3(a) shows the results of a simulation of the objective lens. We have manufactured several prototypes and tested them using a focused electron gun with minimum spot size of $2 \mu \mathrm{m}$.

The objective lens is installed in front of an R4000 $10 \mathrm{kV}$ hemispherical analyzer. The angle acceptance of the input lens of the analyzer is $\pm 7^{\circ}$. This restricts the angle acceptance of the combined objective lens and the analyzer to $\pm 35^{\circ}$. The magnification factor of the input lens is 5, thus the total magnification factor is 25 . To increase the efficiency of the photoelectron acceptance into the analyzer slit, one should minimize blur of the focusing at the exit of the objective lens.

\section{Experimental}

The X-ray spot size was evaluated by the line profile extracted from secondary electron image of a copper mesh obtained by scanning the X-ray beam. The focusing characteristic of the wide acceptance objective lens was evaluated by measuring the

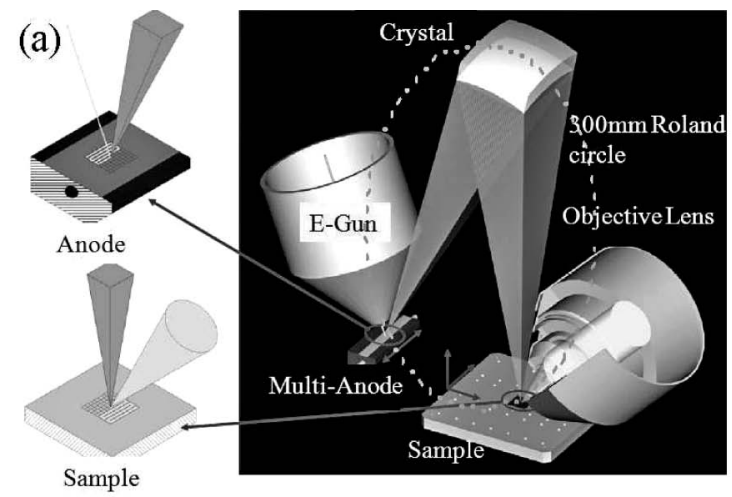

(b) Scanning X-ray SED Image
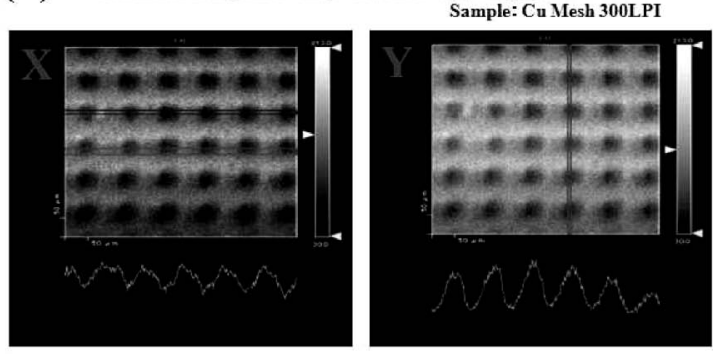

Spatial Resolution

$\mathrm{X}: \underline{9.11 \mu m}$ Y: $\underline{8.37 \mu m}$

Fig. 2 (a) Schematic view of monochromatic $\mathrm{Cr} \mathrm{K}_{\alpha}$ focused X-ray source. A focused electron beam is irradiated on the water cooled $\mathrm{Cr}$ target, and the emitted X-rays are monochromatized and focused on to the sample using a bent crystal monochromator with a $300 \mathrm{~mm}$ Roland circle. (b) The minimum X-ray spot size of $9 \mu \mathrm{m} \times 8 \mu \mathrm{m}$ was achieved as evaluated from the secondary electron image of a copper mesh sample.

focus spot size of the elastic scattered electrons from a gold plate target at the source point of the lens, irradiated by a focused electron beam at $5 \mathrm{keV}$ with minimum spot size of $2 \mu \mathrm{m}$. The focused images of the elastically scattered electrons were detected by a phosphor screen and a CCD camera. Electron optics simulations were performed to guide the determination of the best focus condition. After the performance tests of the X-ray source, it was combined with the R4000 $10 \mathrm{kV}$ analyzer to estimate the performance without objective lens. $\mathrm{Au} 3 \mathrm{~d}$ and $\mathrm{Au}$ valence band spectra were measured. Then the analyzer was retracted to insert the objective lens and the same spectra were measured again. Angle acceptance and angle resolution of the whole system were estimated by measuring angle resolved $\mathrm{Au} 3 \mathrm{~d}_{5 / 2}$ spectrum of a device in which an $\mathrm{Au}$ thin strip was covered with a hemicylindrical multi-slit.

Preliminary measurements were performed at Si-LSI-related samples, such as $\mathrm{SiO}_{2} / \mathrm{Si}(001)$ and $\mathrm{HfO}_{2} / \mathrm{SiO}_{2} / \mathrm{Si}(001)$ gate stack structures, to prove the feasibility of this laboratory HXPS system. Polar-azimuth angle mappings of $\mathrm{Si} 1 \mathrm{~s}$ intensity in $\mathrm{Si}(001)$ samples covered by natural oxide and $4 \mathrm{~nm}$ and $7.5 \mathrm{~nm}$ $\mathrm{SiO}_{2}$ layers were performed to demonstrate the potential of bulk sensitive X-ray photoelectron diffraction (XPD) observations.

\section{Results and Discussion}

Focused spot images of the objective lens and its profile were 
(a) SIMION trace calculation Cross-section (for $1 \mathrm{kV}$ )

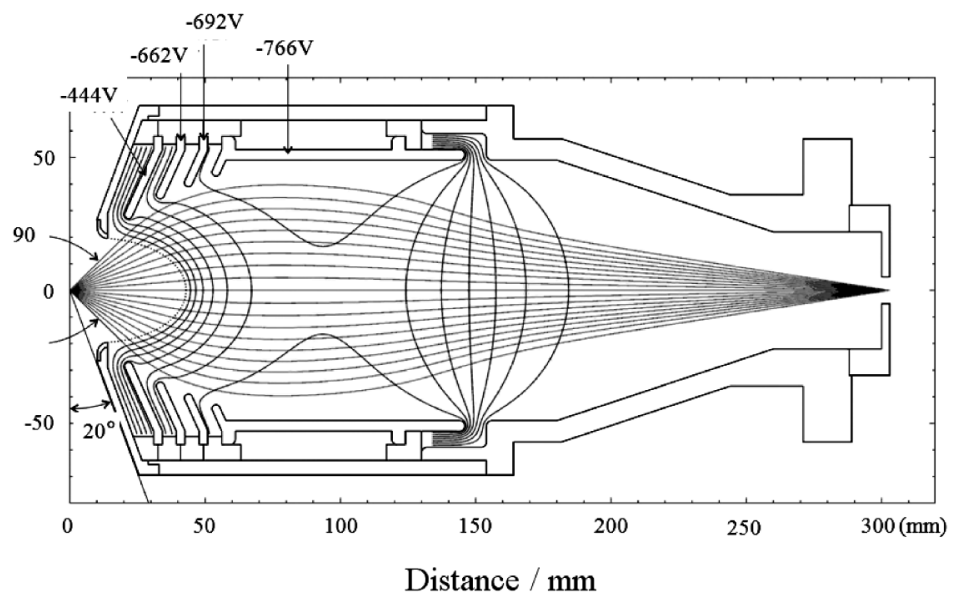

(b)

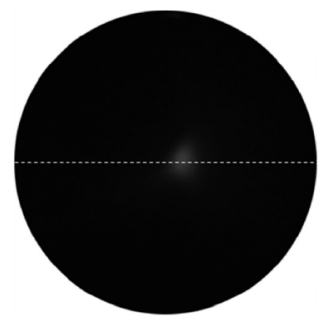

(c)

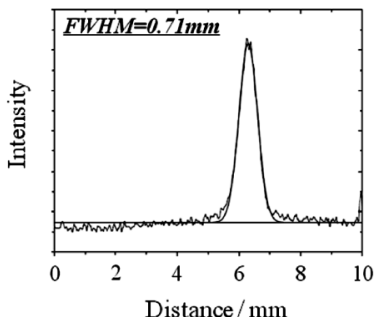

Fig. 3 (a) Structure and electron trajectory simulation of the wide acceptance objective lens. (b) Focused spot at the exit of the wide acceptance objective lens. (c) Cross-section profile of the focused spot (Profile of the image along the line indicated in (b)).

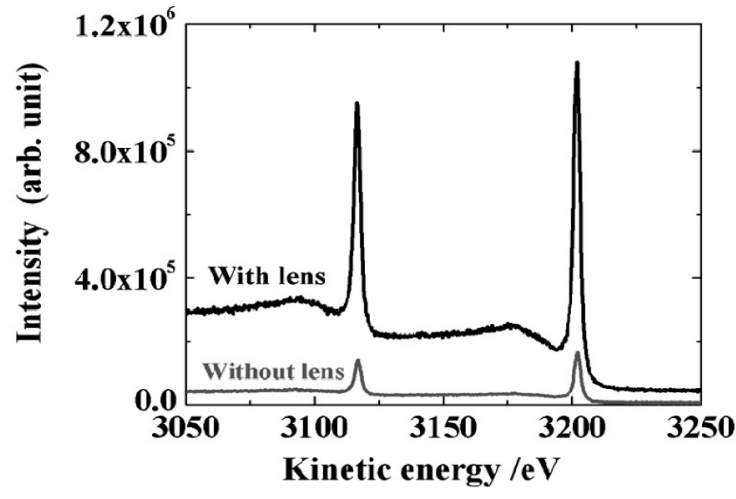

Fig. 4 Au 3d spectra measured with and without the wide acceptance objective lens by $\mathrm{Cr} K_{\alpha}$ excitations of $50 \mathrm{~W}$ and $200 \mu \mathrm{m}$ spot size. These spectra were recorded at $200 \mathrm{eV}$ pass energy and $1.5 \mathrm{~mm}$ slit-width, and the electron take-off-angle was $80^{\circ}$.
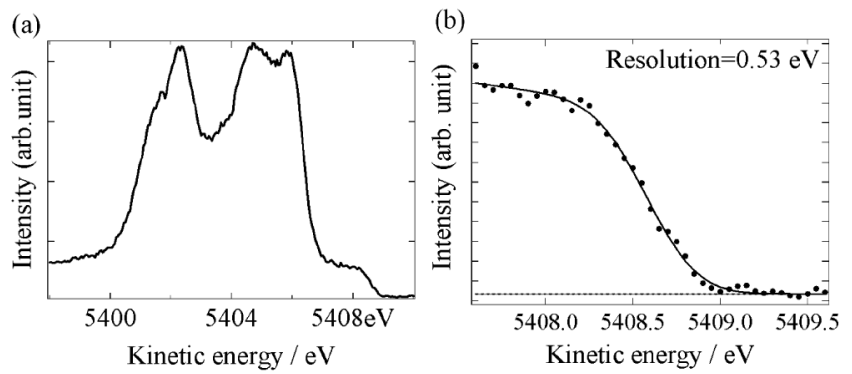

Fig. 5 (a) Valence band spectra of Au plate and (a) Fermi-edge profile. These spectra were recorded at $200 \mathrm{eV}$ pass energy and 0.8 $\mathrm{mm}$ slit-width, and the electron take-off-angle was $85^{\circ}$.

minimized by varying the potentials applied to the lens elements. Examples of minimized a spot image and of its profile for $5 \mathrm{keV}$ incident electrons are shown in Figs. 3(b) and (c). The coincidence between lens parameter sets experimentally determined by this minimization procedure and those obtained (a)

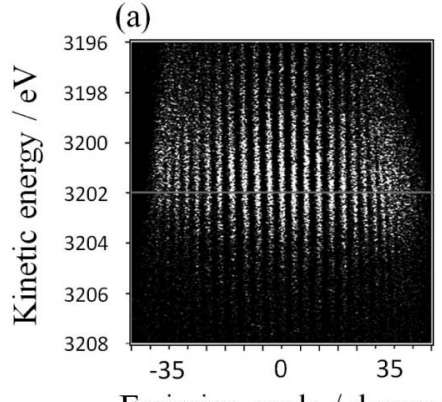

(b)

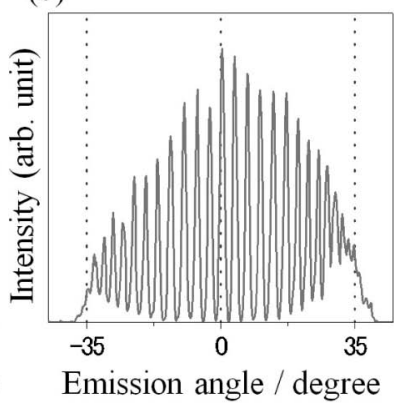

Fig. 6 The angular resolution of the wide acceptance objective lens was tested using a test device composed of an Au plate, in front of which a combination of a long slit and a cylindrical multi-slit is mounted. (a) Image of $\mathrm{Au} 3 \mathrm{~d}_{5 / 2}$ peak recorded on the $2 \mathrm{D}$ detector in the angular mode of the VG Scienta $10 \mathrm{kV}$ analyzer. (b) Profile of the image along the line indicated in (a). $\mathrm{A} \pm 35^{\circ}$ acceptance angle with angular resolution better than $0.5^{\circ}$ is evident in this result. Period of the multi-slit is $2.8^{\circ}$. This spectrum was recorded at $200 \mathrm{eV}$ pass energy and $2.5 \mathrm{~mm}$ slit-width, and the electron take-off-angle was $80^{\circ}$.

by simulation was excellent.

Basic performances of the whole laboratory system were evaluated by $\mathrm{Au} 3 \mathrm{~d}$ and valence band spectra of Au. Figure 4 shows a comparison of $\mathrm{Au} 3 \mathrm{~d}$ spectra recorded using the R4000 $10 \mathrm{kV}$ analyzer with and without the objective lens. The experimental conditions are as follows: X-ray spot size $200 \mu \mathrm{m}$, pass energy $200 \mathrm{eV}$, analyzer entrance slit size $4 \mathrm{~mm}$. The acquisition time for the $\mathrm{Au} 3 \mathrm{~d}$ spectra with the objective lens was 16 min, as shown in Fig. 4. Seven times enhancement of the intensity was affirmed by comparing the intensities of $\mathrm{Au} 3 \mathrm{~d}$ doublet peaks with or without the objective lens. The total resolution of $0.53 \mathrm{eV}$ was verified by $\mathrm{Au}$ Fermi-edge measurements, as shown in Fig. 5. The angular acceptance and angular resolution were evaluated using a testing sample to generate $\mathrm{Au} 3 \mathrm{~d}_{5 / 2}$ photoelectrons through a hemicylindermulti-slit for collimation on Au thin strips. As shown in Fig. 6, an acceptance angle of $\pm 35^{\circ}$ and a resolution of less than $0.5^{\circ}$ were achieved. 


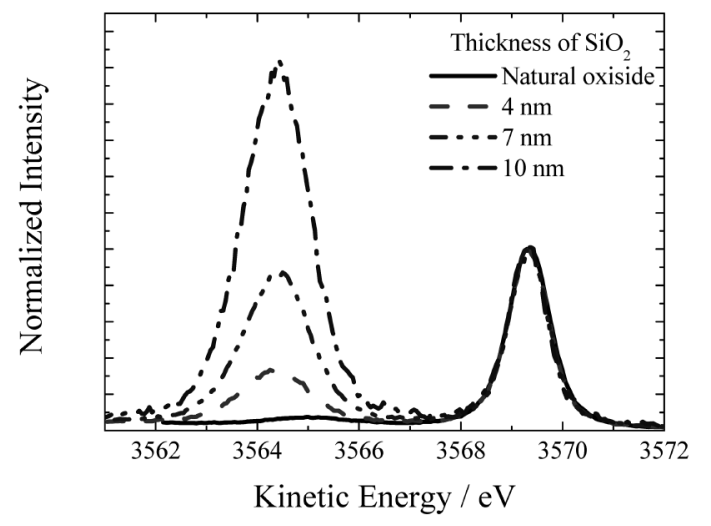

Fig. 7 Si 1s spectra of a $\mathrm{Si}(001)$ substrate covered with $\mathrm{SiO}_{2}$ layers of various thicknesses. These spectra were recorded at $200 \mathrm{eV}$ pass energy and $0.8 \mathrm{~mm}$ slit-width, and the electron take-off-angle was $80^{\circ}$.
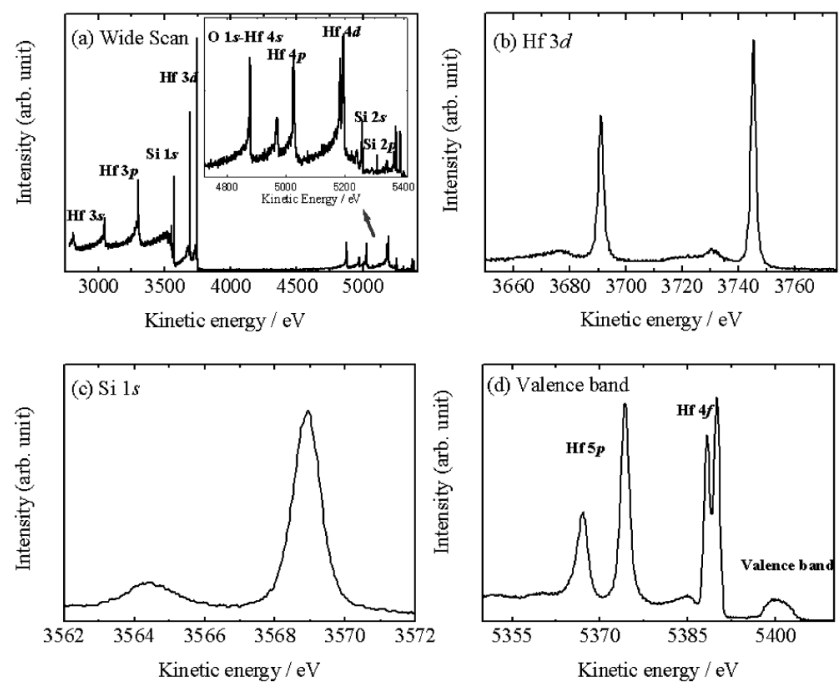

Fig. 8 (a) Wide scan, (b) $\mathrm{Hf} 3 \mathrm{~d}$, (c) $\mathrm{Si} 1$ s, and (d) valence band spectra of the $\mathrm{HfO}_{2}(4 \mathrm{~nm}) / \mathrm{SiO}_{2}(1 \mathrm{~nm}) / \mathrm{Si}(001)$ multilayer structure. These spectra were recorded at $200 \mathrm{eV}$ pass energy and $0.8 \mathrm{~mm}$ slit-width, and the electron take-off-angle was $80^{\circ}$.

As an illustration of the large information depth characteristics of our system, the $\mathrm{Si} 1 \mathrm{~s}$ spectra at the TOA from the surface plane of $80^{\circ}$ from $\mathrm{SiO}_{2}(0-10 \mathrm{~nm}) / \mathrm{Si}$ samples are shown in Fig. 7. These results clearly explain that HXPS can detect deeper regions comparing to conventional XPS due to weaker inelastic scattering for higher kinetic energy electrons. Thus the developed laboratory HXPS system opens up unique possibilities for buried layer measurements of thin film samples at laboratories without surface cleaning prior to the measurements.

The large information depth of HXPS already exceeds the thickness of the multilayers used in advanced devices such as Si-ULSI, thus this laboratory's HXPS system has the potential to be a useful tool in the research of semiconductor nano-devices. As examples, we demonstrate two samples of multilayer thin films on silicone substrates for illustrating the abilities of the HXPS system in this field.

Figure 8 shows wide scan, Si 1s, Hf $3 \mathrm{~d}$, and valence band spectra at the TOA of $80^{\circ}$ measured from the surface of the $\mathrm{HfO}_{2}(4 \mathrm{~nm}) / \mathrm{SiO}(1 \mathrm{~nm}) / \mathrm{Si}(001)$ multilayer sample. Acquisition (a)
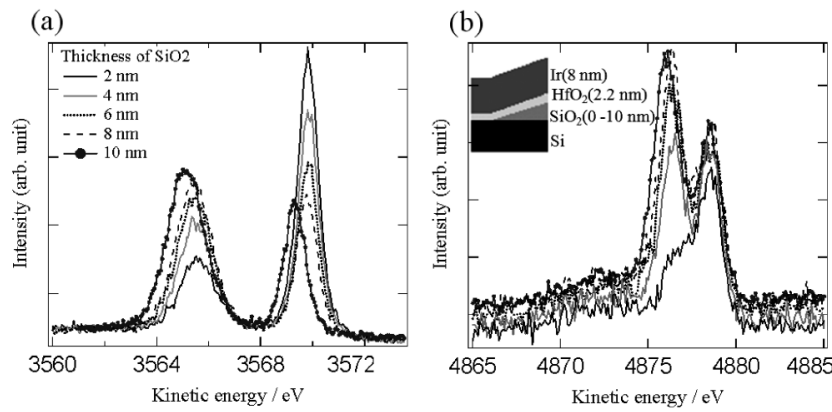

(c)

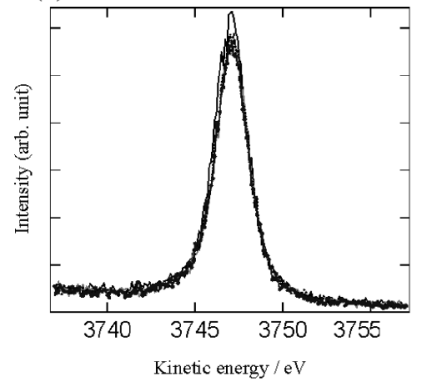

(d)

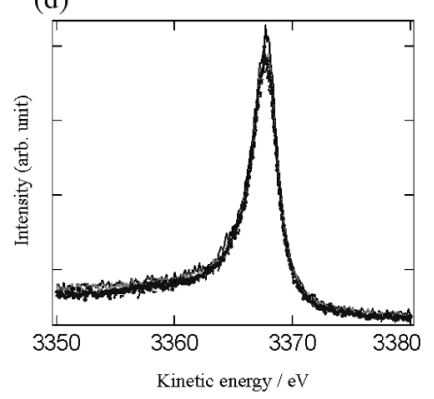

Fig. 9 (a) Si 1s, (b) O $1 \mathrm{~s}$, (c) $\mathrm{Hf} 3 \mathrm{~d}_{5 / 2}$ and (d) Ir $3 \mathrm{~d}_{5 / 2}$ photoelectron spectra obtained from the $\mathrm{Ir}(8 \mathrm{~nm}) / \mathrm{HfO}_{2}(2.2 \mathrm{~nm}) / \mathrm{SiO}_{2}(0-10 \mathrm{~nm}) / \mathrm{Si}$ multilayer structure. The inset schematically represents the structure of the $\mathrm{Ir} / \mathrm{HfO}_{2} / \mathrm{SiO}_{2} / \mathrm{Si}$ multilayer. These spectra were recorded at $200 \mathrm{eV}$ pass energy and $0.8 \mathrm{~mm}$ slit-width, and the electron take-off-angle was $80^{\circ}$.

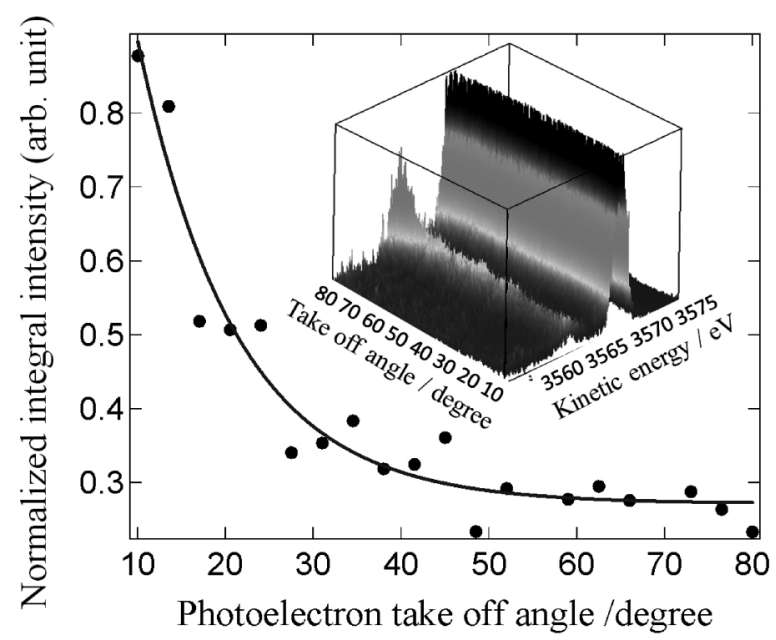

Fig. $10 \mathrm{Si}$ 1s spectra of $\mathrm{SiO}_{2}(4 \mathrm{~nm}) / \mathrm{Si}$ structure measured in angular mode. The normalized integral intensity of $\mathrm{SiO}_{2}$ component is shown as a function of the take-off angle. These spectra were recorded at $200 \mathrm{eV}$ pass energy and $0.8 \mathrm{~mm}$ slit-width.

times of $17 \mathrm{~min}, 5 \mathrm{~min}, 10 \mathrm{~min}$, and $12 \mathrm{~h}$ included enough signal to noise $(S / N)$ to obtain wide scan (a), Hf 3d (b), Si 1s (c), and valence band (d) spectra, respectively.

The second example is a result in an $\mathrm{Ir}(8 \mathrm{~nm}) / \mathrm{HfO}_{2}$ $(2.2 \mathrm{~nm}) /$ wedge-shaped $\mathrm{SiO}_{2}(0-10 \mathrm{~nm}) / \mathrm{Si}(100)$ sample at the TOA of $80^{\circ}$ supplied by Abe et al., ${ }^{8}$ shown in Fig. 9. The intensity ratio of $\mathrm{O} 1 \mathrm{~s}$ components which are to be identified as $\mathrm{SiO}_{2}$ at $4876 \mathrm{eV}$ and $\mathrm{HfO}_{2}$ at $4878 \mathrm{eV}$ varies with $\mathrm{SiO}_{2}$ thickness. Si 1s (both substrate and oxides) peak shapes also depend on the $\mathrm{SiO}_{2}$ thickness. No distinguishable variation with 

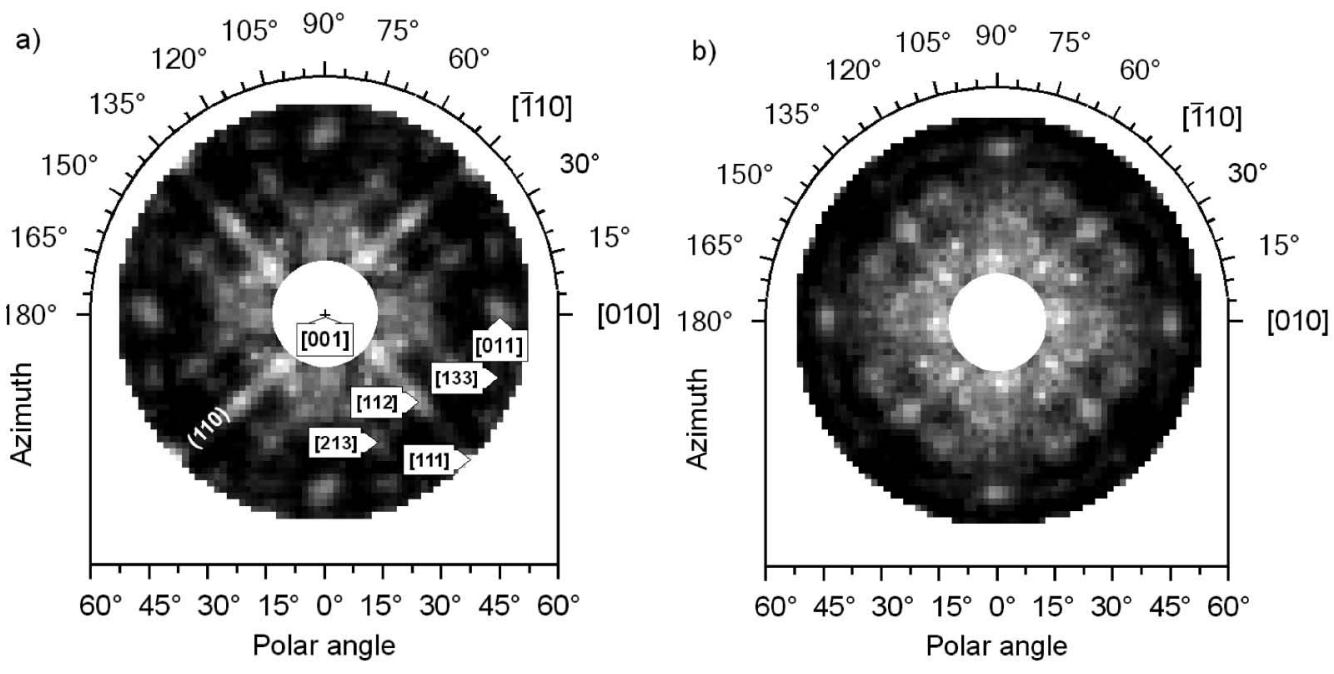

Fig. 11 Photoelectron diffraction pattern of the $\mathrm{Si} 1$ s peak from $\mathrm{Si}(100)$ covered by natural oxide (a) and $4 \mathrm{~nm}$-thick $\mathrm{SiO}_{2}$ overlayer (b). The kinetic energy of the photoelectrons was $3569 \mathrm{eV}$. A four-fold symmetry operation is applied. Some low-index crystalline directions are labelled in (a). These spectra were recorded at $200 \mathrm{eV}$ pass energy and $0.8 \mathrm{~mm}$ slit-width.

$\mathrm{SiO}_{2}$ thickness in $\mathrm{Hf} 3 \mathrm{~d}_{5 / 2}$ and Ir $3 \mathrm{~d}_{5 / 2}$ was recognized. Detailed analyses and discussion will be given elsewhere. The $\mathrm{Si} 1 \mathrm{~s}$ photoelectrons from the substrate at kinetic energy of $3569 \mathrm{eV}$ are still observed through the $20 \mathrm{~nm}$ thickness overlayers.

The wide acceptance angle objective lens allow us to get information from the surface and bulk region at the same time. In other words, angle resolved HXPS spectra can be measured in the TOA range of $70^{\circ}$ without tilting the sample. A typical TOA dependence of $\mathrm{SiO}_{2}(4 \mathrm{~nm}) / \mathrm{Si}(001) \mathrm{Si} 1 \mathrm{~s}$ spectra in angular resolution mode was measured. Figure 10 shows the TOA dependence of substrate and $\mathrm{SiO}_{2} \mathrm{Si}$ 1s peaks normalized at the substrate peak intensity. Normalized intensities of the oxidized states of Si 1s spectra changed in accordance with the TOA, which corresponds to the thickness of the $\mathrm{SiO}_{2}$ layer. The acquisition time to obtain TOA dependence in the TOA range of $70^{\circ}$ of $\mathrm{Si} 1 \mathrm{~s}$ spectra is $\mathrm{ca}$. $60 \mathrm{~min}$, with sufficient $S / N$ to analyze thickness of $\mathrm{SiO}_{2}$ at the present stage.

The last application example is an observation of bulk X-ray photoelectron diffraction (XPD). XPD is one of the useful techniques for investigating local atomic surface structure., ${ }^{9,10}$ The hard X-ray regime of the XPD (HXPD), with electron kinetic energies $\geq 2 \mathrm{keV}$, is supposed to open up additional possibilities in accessing truly bulk properties of a wide variety of materials. ${ }^{11,12}$ A detailed description of the expected benefits of HXPD is given in Ref. 13. The HXPD measurements were performed at the $\mathrm{Si} 1 \mathrm{~s}$ core level photoelectrons, at kinetic energy $3569 \mathrm{eV}$, from $\mathrm{Si}(001)$ substrate covered by thermally grown $\mathrm{SiO}_{2}$ overlayers with different thicknesses. Figure 11 displays HXPD patterns obtained from $\mathrm{Si}(100)$ covered by a natural oxide (Fig. 11(a)) and a 4 nm-thick silicon dioxide (Fig. 11(b)) overlayer. The HXPD patterns were measured with an angular resolution of $0.35^{\circ}$ in the polar direction and with $3^{\circ}$ step in the azimuthal direction. The azimuthal angle was scanned in the range of over $90^{\circ}$. The rotational symmetry operation was applied, considering the symmetry of the crystal. The measurement time required for one pattern was approximately $30 \mathrm{~h}$.

Theoretical studies predicted that the electron-atom scattering proces at high kinetic energies produce a strong enhancement of the intensity in the forward scattering process. ${ }^{11}$ Such

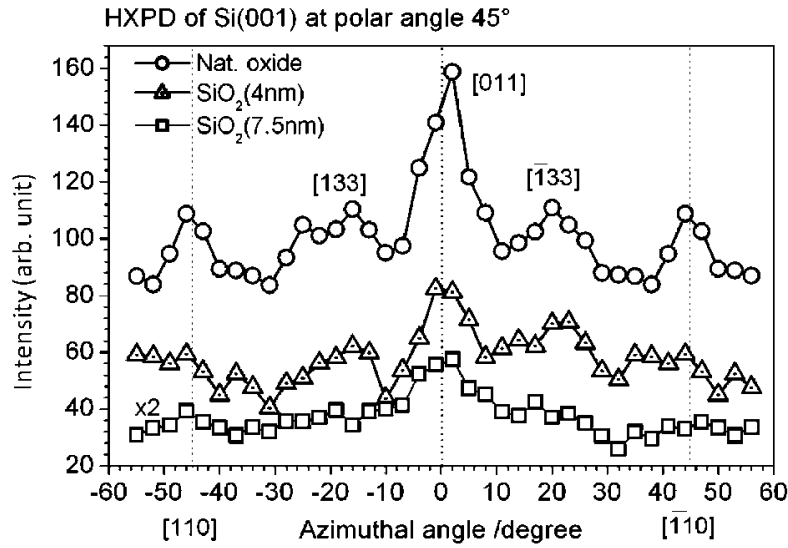

Fig. 12 The intensity of the $\mathrm{Si} 1 \mathrm{~s}$ peak from $\mathrm{Si}(001)$ as a function of azimuthal angle measured at a polar angle of $45^{\circ}$. The curves correspond to the diffraction from the $\mathrm{Si}(001)$ substrate covered by $7.5 \mathrm{~nm}$ (bottom), $4 \mathrm{~nm}$ (middle) thick silicon dioxide overlayer and natural oxide overlayer (top). The intensities in the bottom curve are multiplied by the factor 2 . These spectra were recorded at $200 \mathrm{eV}$ pass energy and $0.8 \mathrm{~mm}$ slit-width.

forward-scattering effects should provide real-space information on the directions of near-neighbor bonds and low-index axes. The positions of the main spots in Fig. 11 are in good agreement with low-index directions in the silicon crystal structure.

Other significant features in the HXPD pattern in Fig. 11(a) are lines of enhanced intensity along the [110] and [-110] directions. Their origin comes from a forward-focusing effect in the (110) plane and from long-order Bragg diffraction, which creates the well-known Kikuchi bands from (110) atomic planes. $^{14}$

Figure 12 shows the intensity of $\mathrm{Si} 1$ s peak from the $\mathrm{Si}(001)$ substrate as a function of azimuthal angle measured at polar angle $45^{\circ}$. The width of modulation by photoelectron diffraction seems to disappear with increasing of the oxide thickness, due to the elastic scattering process in this amorphous overlayer. 
Nevertheless, the diffraction is still observed even through the $\mathrm{SiO}_{2}$ overlayer has a thickness of more than $7.5 \mathrm{~nm}$. The rapid decrease of the peak at azimuth $[-110]$ is connected with the diminishing effect of the Bragg diffraction from (110) planes. The intensity of the Kikuchi band depends on the depth from which the signal is received. Diffraction patterns at selected kinetic energy values are formed from thicknesses of the order of the inelastic mean free path, that is, about $6.7 \mathrm{~nm}$ in the case of clean silicon surface..$^{15}$ The information depth in a silicon crystal covered by a silicon dioxide overlayer is lower than that of natural oxide layers, and that causes the decrease of the intensity in [-110] direction.

\section{Conclusions}

In conclusion, the laboratory HXPS system which consists of a $\mathrm{Cr} \mathrm{K}_{\alpha}$ monochromatic source, a wide acceptance objective lens and a high energy analyzer was proved to be a promising tool for the investigation of bulk and thin solid film materials. The applicability of the system to detect the depth information of layered materials more than $10 \mathrm{~nm}$ thicknesses was confirmed. Distinguishable XPD modulation of the crystalline substrate was observed even in $\mathrm{Si}(001)$ substrates with $\mathrm{SiO}_{2}$ overlayers thicker than $4 \mathrm{~nm}$. It is expected to be a useful probe for the local structure around the doped impurity atoms accompanied by local distortion of crystal lattices. On the other hand, it may also cause a certain difficulty to the depth profile analysis: e.g., the intensity of angle-dependence for single crsytal is affected by diffraction. Although the present results are still preliminary, the laboratory HXPS system developed was proved to be promising for investigations of electronic structures and chemical states in various bulk materials, nano-scale thin films, multilayers, and nano clusters, and their interfaces with practical throughput. Further efforts are being undertaken to improve the performance of the system.

\section{Acknowledgements}

The author is grateful to Drs. E. Ikenaga, M. Machida, and J. Y. Son of JASRI for their cooperation and advice in the development. We are thankful to Dr. N. Miyata, Dr. Y. Abe, and Prof. H. Nohira for providing samples. This work is supported by SENTAN, JST.

\section{References}

1. K. Kobayashi, Nucl. Instrum. Methods Phys. Res., Sect. A, 2009, 604, 8.

2. K. Kobayashi, M. Yabashi, Y. Takata, T. Tokushima, S. Shin, K. Tamasaku, D. Miwa, T. Ishikawa, H. Nohira, T. Hattori, Y. Sugita, O. Nakatsuka, A. Sakai, and S. Zaima, Appl. Phys. Lett., 2003, 83, 1005.

3. The electron inelastic-mean-free-paths were estimated using NIST 111 Standard Reference Database 71, "NIST Electron Inelastic-Mean-112 Free-Path Database: Ver. 1.1" and references therein. It is distributed via the Web site 113, http://www.nist.gov/srd/nist71.htm.

4. Y. Takata, K. Tamasaku, T. Tokushima, D. Miwa, S. Shin, T. Ishikawa, M. Yabashi, K. Kobayashi, J. J. Kim, T. Yao, T. Yamamoto, M. Arita, H. Namatame, and M. Taniguchi, Appl. Phys. Lett., 2004, 84, 4310.

5. J. J. Yeh and I. Lindau, Atom. Data Nucl. Data Tables, 1985, 32, 1.

6. H. Matsuda, H. Daimon, M. Kato, and M. Kudo, Phys. Rev. $E$, 2005, 71, 066503 .

7. H. Matsuda and H. Daimon, Japan Patent: PCT/jp2004/016602, Japan 2004-208926.

8. Y. Abe, N. Miyata, E. Ikenaga, H. Suzuki, K. Kitamura, S. Igarashi, and H. Nohira, Jpn. J. Appl. Phys., 2009, 48, 041201

9. C. S. Fadley, Phys. Scr., 1987, T17, 39.

10. C. S. Fadley, J. Electron. Spectrosc., 1994, 68, 19.

11. C. S. Fadley, Nucl. Instrum. Methods Phys. Res., Sect. A, 2005, 547, 24.

12. C. S. Fadley, Nucl. Instrum. Methods Phys. Res., Sect. A, 2009, 601, 8 .

13. A. Winkelmann, New J. Phys., 2008, 10, 113002.

14. M. V. Gomoyunova, I. I. Pronin, N. S. Faradzhev, and D. A. Valdatsev, Phys. Solid State, 1999, 41, 411.

15. S. Tanuma, C. J. Powell, and D. R. Penn, Surf. Interface Anal., 1993, 21, 165. 